\title{
Cenpestao
}

\section{Redes de colaboração científica em Educação Física: comparação entre a subárea Biodinâmica do Movimento e a subárea Sociocultural e Pedagógica}

\author{
Felipe Ferreira Barros Carneiro \\ Doutor; Instituto Federal de Educação, Ciência e Tecnologia do Espírito Santo, Santa Maria de Jetibá, ES, \\ Brasil; \\ felipe.carneiro@ifes.edu.br \\ Amarílio Ferreira Neto \\ Doutor; Universidade Federal do Espírito Santo, Vitória, ES, Brasil; \\ amariliovix@gmail.com \\ Wagner dos Santos \\ Doutor; Universidade Federal do Espírito Santo, Vitória, ES, Brasil; \\ wagnercefd@gmail.com
}

\begin{abstract}
Resumo: O estudo objetiva investigar, caracterizar e analisar as redes de colaboração científica existentes na área de Educação Física, de maneira a compreender o desenvolvimento histórico das redes da subárea Biodinâmica do Movimento e da subárea Sociocultural e Pedagógica, estabelecendo uma comparação entre as suas características e entre seus impactos na comunicação científica. Utiliza como fonte dez periódicos científicos de cada subárea. Para a produção e manipulação das redes, foram combinados os softwares Endnote X7.5 (produção do banco de dados), VOSviewer 1.6.10 (elaboração da rede primária) e Gephi 0.92 por meio do algoritmo Fruchterman-Reingold. Concluise que as duas subáreas têm se desenvolvido de maneira fragmentada, mas por meio de redes do tipo small worlds.
\end{abstract}

Palavras-chave: Educação Física. Redes de colaboração. Cientometria.

\section{Introdução}

De acordo com Curiel (2015), as redes sociais formam parte das rotinas de qualquer meio de comunicação. Nesses termos, as redes são uma maneira de representar as cooperações afetivas ou profissionais entre integrantes que se conectam horizontalmente (PINTO; GONZALES-AGUILAR, 2014). Cabe ressaltar que o elevado crescimento da produção científica mundial tem sido acompanhado pelo aumento da colaboração entre pesquisadores e, por consequência, pela ampliação do interesse da comunidade científica em compreender essas redes de colaboração (HADDAD; MENA-CHALCO, 2016; 
HILÁRIO; GRÁCIO; GUIMARÃES, 2018; SIDONE; SOUSA; FONTENELE, 2019).

Estudar a colaboração científica de um campo de conhecimento específico possibilita observar resultados que permitam orientar a gestão da pesquisa em uma organização e/ou área da ciência. O trabalho colaborativo, segundo Katz e Martin (1997), não requer unicamente o conhecimento técnico e científico, mas inclui, também, habilidades de relacionamento e gerenciamento interpessoal. Esse processo pode ser definido como "[...] dois ou mais cientistas trabalhando juntos em um projeto de pesquisa, compartilhando recursos intelectuais, econômicos e/ou físicos." (VANZ; STUMP, 2010, p. 44).

Camargo (2016) afirma que a sociedade contemporânea se encontra em uma imensa rede. Assim, a ciência é praticada e produzida coletivamente pela ligação de várias competências e pela associação dos níveis sociais, técnicos e financeiros. Nesses termos, conforme afirmam Digiampietri e colaboradores (2014), as ligações intelectuais entre cientistas se estabelecem por meio de relações sociais, de modo que, talvez, mais importante que o financiamento da ciência em polos, ainda em processo de consolidação, é a inserção efetiva desses pesquisadores nas redes de colaboração acadêmica.

Partindo dessa premissa, podemos perceber que a atuação conjunta dos pesquisadores torna-se um elemento fundamental para o desenvolvimento de projetos coletivos, para a ampliação da produtividade científica e do impacto e, por consequência, para a obtenção de avanços desejáveis para os distintos campos do conhecimento, além de potencializar o processo de internacionalização da ciência por meio da inserção dos pesquisadores em redes de colaboração mundiais.

De acordo com Todeschini e Baccini (2016), as análises das redes de colaboração estão baseadas nas "teorias dos grafos". Um grafo é um tipo de representação matemática gráfica no qual, para qualquer conjunto $G, G=\{V$, E\} - em que $V$ representa um conjunto de elementos não ordenados denominados nós, e E é um subconjunto que une os elementos de G, chamados arestas. 


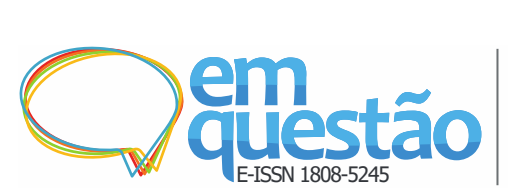

No caso das redes de colaboração científica, os nós representam os autores, as arestas indicam as formas de colaboração existentes entre os cientistas de um campo e a espessura das arestas representa a frequência com que os pesquisadores colaboram (TODESCHINI; BACCINI, 2016).

A área de Educação Física faz parte da grande área da Saúde e está organizada, juntamente com a Fisioterapia, a Fonoaudiologia e a Terapia Ocupacional, em uma de suas ramificações na denominada Área 21,

De acordo com o documento de área da Coordenação de Aperfeiçoamento de Pessoal de Nível Superior (CAPES) CAPES (2016), a área de Educação Física é demarcada pela multidisciplinaridade, indicando tanto a aproximação do campo com as Ciências Naturais, que vem sendo tratada academicamente como a subárea da Biodinâmica do Movimento (SBM), quanto a aproximação com as Humanidades, que são as práticas denominadas como subárea Sociocultural e Pedagógica (SSCP).

Sobre a SBM, Tani (1989) já destacava que os estudos dessa natureza normalmente estão voltados para os mecanismos de sustentação para o movimento (bioquímica e fisiológica) e de organização motora em seus aspectos físicos (biomecânica), o que, nos termos de Bracht (2003), são conhecimentos que, solidamente, vêm se ancorando nas Ciências Biológicas ou, de maneira mais ampla, nas Ciências Naturais (KOKUBUN, 2004).

Já a SSCP, para Manoel e Carvalho (2011, p. 392), “[...] trata de temas como esporte, práticas corporais e atividade física [...]”. Além disso, “[...] investiga questões relativas à formação de professores, ao desenvolvimento curricular, aos métodos de ensino [...]" (MANOEL; CARVALHO, 2011, p. 392). Desse modo, as práticas científicas dessa subárea estão ligadas a conhecimentos e abordagens da área de Ciências Humanas e Sociais, especialmente, por meio de suas teorias e matrizes filosóficas (CARNEIRO; FERREIRA NETO; SANTOS, 2015).

Essa condição coloca a área de Educação Física em uma zona de fronteira entre as características expostas por Larivière e outros (2006) no que diz respeito à aceitação de indicadores bibliométricos, na medida em que o 


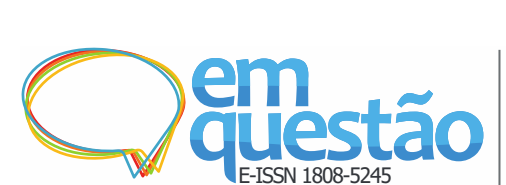

campo reúne em seu interior práticas científicas que se aproximam tanto das Ciências Naturais, quanto das Ciências Humanas.

Desse modo, o objetivo deste trabalho é investigar, caracterizar e analisar as redes de colaboração científica existentes na área de Educação Física, de maneira a compreender o desenvolvimento histórico das redes de cada subárea, estabelecendo uma comparação das características das redes da SBM e da SSCP e de seus impactos na comunicação científica.

\section{Procedimentos metodológicos}

Os dados foram construídos tendo como fonte periódicos científicos do campo de Educação Física. O corpus de análise foi elaborado tomando como referência os periódicos mais utilizados como alvo para escoamento da produção científica pelos Bolsistas de Produtividade em Pesquisa da área de Educação Física, que foram separados com base em seus alinhamentos epistemológicos entre a SBM e a SSCP.

As publicações desses pesquisadores foram utilizadas como critério de seleção dos periódicos por entendermos que esse grupo pertence a uma parcela da comunidade acadêmica reconhecida nacionalmente por seus pares e pelas agências de fomento. Parte-se do pressuposto de que suas produções circulam nos periódicos mais qualificados no campo científico no cenário brasileiro e internacional.

No ano de 2017, existiam 91 bolsistas de produtividade no campo de Educação Física. A dispersão desses pesquisadores nos níveis de bolsa encontrase da seguinte forma: sete no nível 1A; cinco no nível 1B; cinco no nível 1C; 21 no nível 1D; 52 no nível 2; e um bolsista sênior ${ }^{1}$.

Buscaram-se nos currículos Lattes dos pesquisadores bolsistas todas as publicações feitas em periódicos científicos no período em que concorreram aos editais e foram contemplados com a bolsa de produtividade em pesquisa na área de Educação Física. Assim, foram catalogados os dez anos das publicações dos bolsistas nível 1 e os cinco anos dos bolsistas nível 2 conforme critérios previstos na RN-028/2015, perfazendo um total de 6437 registros de publicações recuperadas entre os anos de 2001 a 2015. 


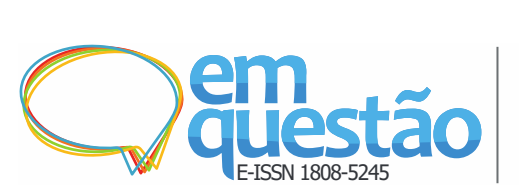

Os dados foram parametrizados a partir da média ponderada entre o número de vezes que um periódico aparece e o quantitativo de pesquisadores de produtividade presentes naquela categoria. O quociente desse processo foi multiplicado por um peso atribuído a cada um dos níveis de bolsa de produtividade conforme seu grau de relevância ${ }^{2}$.

Os periódicos foram organizados em uma listagem única, na qual os valores que eles receberam em cada estrato de bolsista de produtividade foram somados e ordenados de forma decrescente. Desse modo, foi possível identificar os periódicos mais relevantes para esses pesquisadores, o que pode ser considerado como representativo do campo científico, dados a contribuição e o reconhecimento desses pesquisadores na área de Educação Física.

Foram selecionados os periódicos que atenderam aos seguintes critérios: compor o primeiro percentil do ranking de periódicos utilizados pelos bolsistas de produtividade em pesquisa e estar ranqueados entre os níveis A1 e B2 no webqualis no ano de 2017, indicando a relevância dos periódicos selecionados às exigências atuais do campo científico.

Assim, os periódicos selecionados foram:

a) na SBM: Revista Brasileira de Medicina do Esporte (RBME), Journal of Strength and Conditioning Research (JSCR), Revista Brasileira de Cineantropometria \& Desempenho Humano (RBCDH), Revista Brasileira de Educação Física e Esporte (REBEFE), Revista Brasileira de Atividade Física e Saúde (RBAFS), Motriz, Caderno de Saúde Pública (CSP), (IJSM) e Revista da Educação Física da Universidade Estadual de Maringá (REF/UEM);

b) na SSCP: REBEFE, Movimento, Revista Brasileira de Ciências do Esporte (RBCE), Motriz, REF/UEM, Pensar a Prática, Licere, Revista Brasileira de Ciência e Movimento (RBCM) e Motrivivência.

Em razão da mudança do período de avaliação da CAPES de três para quatro anos, os artigos selecionados estão contidos no conjunto de 18784 documentos publicados pelos dez periódicos de cada subárea entre os anos de 2005 e 2016, mantendo-se, assim, intervalos de quatro anos. Os artigos foram 


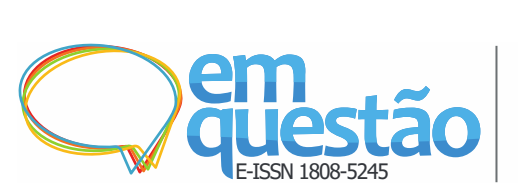

selecionados por amostragem calculada por meio do modelo matemático de Luiz e Magnanini (2009), utilizando os seguintes parâmetros: confiança 95\%, erro amostral $5 \%$.

Assim, o tamanho mínimo calculado foi de 559 artigos, sendo 279 para cada subárea. Porém, uma vez que as análises foram estratificadas em três períodos - 2005-2008; 2009-2012; 2013-2016 - para facilitar o planejamento, optou-se por adotar 300 artigos por subárea, totalizando 600 artigos, que foram selecionados de maneira aleatória entre os números dos periódicos e pesquisas neles veiculadas entre 2005 e 2016.

O processo de seleção aleatória dos artigos tomou como base o princípio de "amostragem sistemática", definida por Torres, Magnanini e Luiz (2009), uma vez que os periódicos mantêm uma organização interna que já dispõe de ordenamentos meticulosos.

A partir dessa delimitação, utilizou-se a tabela de números aleatórios (TORRES; MAGNANINI; LUIZ, 2009) para selecionar o artigo inicial referente a cada ano dos periódicos. Após essa definição, foi estabelecido um contador numérico por periódico em cada ano, tendo como referência a razão entre o total de artigos publicados naquele ano em cada periódico e o número de fascículos por eles veiculados.

As redes de colaboração científica foram produzidas combinando três softwares: Endnote X7.5, VOSviewer 1.6.10 e Gephi 0.92.

Primeiro, foi elaborado dois bancos de dados no Endnote, cada um com os 300 artigos de cada subárea, de modo a recuperar e sistematizar as informações sobre ano, autoria e periódicos de circulação. Essas informações foram exportadas para o VOSviewer, no qual foi gerada a primeira rede.

Após análise preliminar, foram identificados autores em duplicidade. Assim, foi elaborado um thesaurus para duplicar as autorias, permitindo a normatização dos autores. Na sequência, as redes foram tratadas e manipuladas no Gephi, por meio do algoritmo de dispersão Fruchterman-Reingold. Esse é um algoritmo de representação baseado em força direcional que leva em consideração o peso das arestas como variável de atração e repulsão entre os nós (FRUCHTERMAN; REINGOLD, 1991). 


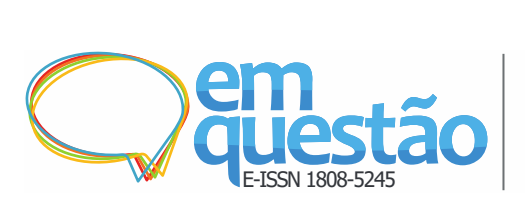

As métricas de análise de rede utilizadas foram:

a) grau médio: número médio de conexões (arestas) dos nós da rede;

b) grau ponderado médio: número médio de conexões (arestas) dos nós da rede ponderado pelo peso atribuído às arestas;

c) densidade da rede: mostra o quanto a rede está próxima de ser completa;

d) componentes conectados;

e) diâmetro da rede: é a menor distância entre os dois nós mais distantes da rede;

f) coeficiente de clusterização médio: indica como os nós estão conectados com sua vizinhança;

g) coeficiente médio de caminho: distância média de grafo entre todos os pares de nós;

h) análise topográfica de componente gigante.

Os grafos de evolução das redes foram elaborados utilizando o filtro de partição de score, selecionando os anos de referência em que os autores publicaram os artigos de acordo com os períodos de 2005-2008, 2009-2012 e 2013-2016, bem como no crescimento acumulado das redes ocorrido entre os seguimentos de 2005-2008, 2005-2012 e 2005-2016.

\section{Resultados e discussão}

As características das redes de colaboração da SBM e da SSCP encontram-se expressas, respectivamente, nas Figuras 1 e 2, nas quais são apresentados os grafos compondo as relações estabelecidas entre os autores nas duas subáreas.

As métricas relativas a essas figuras encontram-se na Tabela 1, na qual estão reunidas as informações referentes aos tópicos analisados nesta seção, de modo a permitir a comparação das redes de colaboração científica das duas subáreas. 
Figura 1 - Grafo de colaboração científica entre pesquisadores da SBM no período de 2005 a 2016

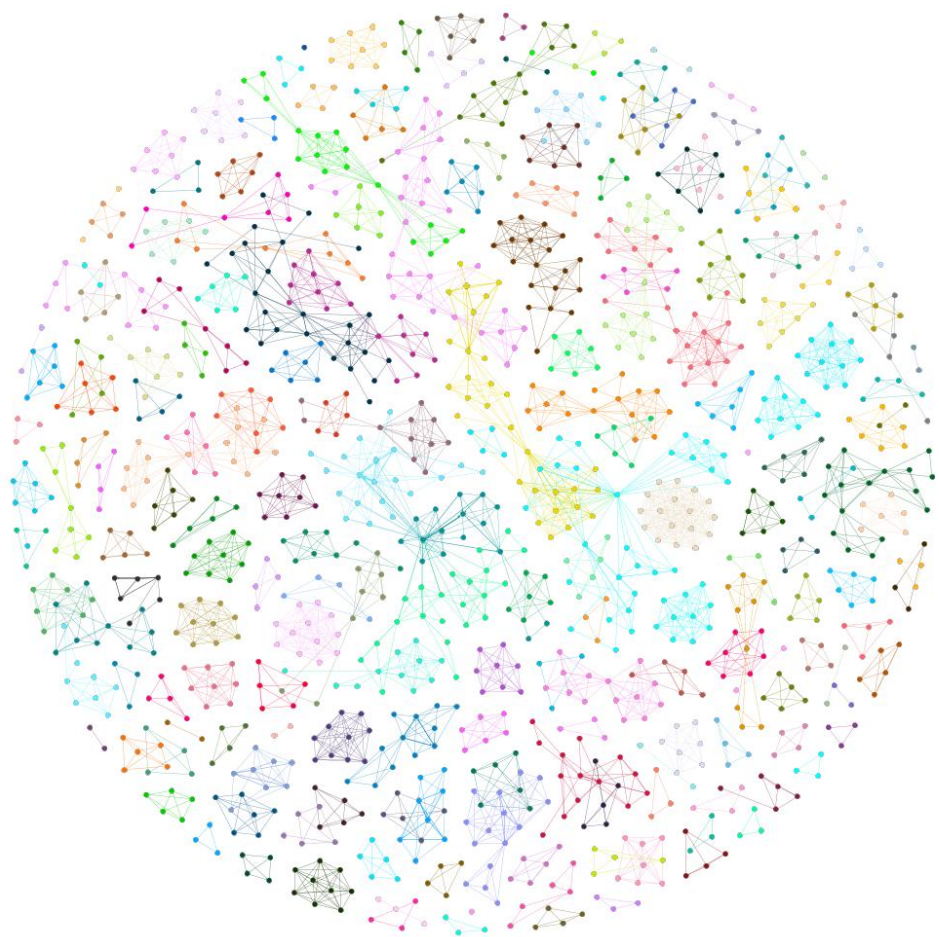

Fonte: Dados da pesquisa.

Figura 2 - Grafo de colaboração científica entre os pesquisadores da SSCP no período de 2005 a 2016

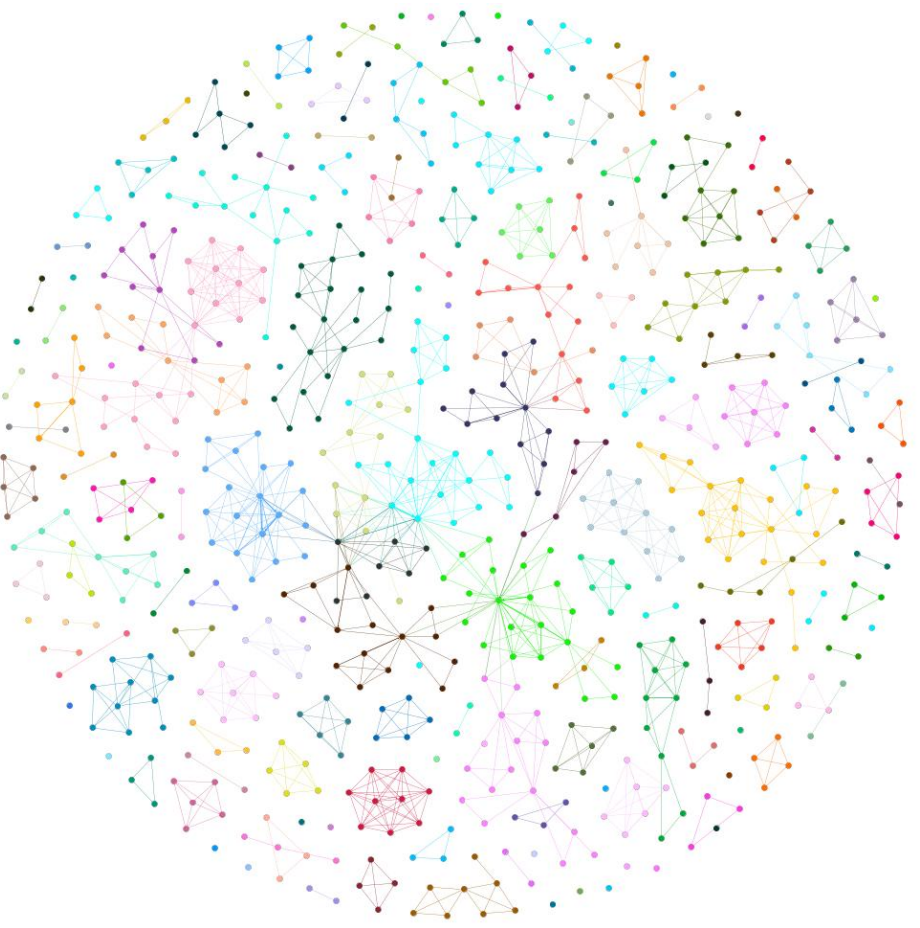

Fonte: Dados da pesquisa. 
Tabela 1 - Características das redes de colaboração científica na SBM e na SSCP no período de 2005 a 2016

\begin{tabular}{lcc}
\multicolumn{1}{c}{ Indicadores } & SBM & SSCP \\
\hline Número de nós & 1279 & 703 \\
Número de arestas & 3347 & 1202 \\
Grau médio & 2,62 & 1,71 \\
Grau ponderado médio & 2,7 & 1,77 \\
Densidade da rede & 0,004 & 0,005 \\
Componentes conectados & 195 & 161 \\
Diâmetro da rede & 6 & 10 \\
Coeficiente de clusterização médio & 0,961 & 0,907 \\
Coeficiente médio de caminho & 2,048 & 3,949 \\
Número de clusters & 199 & 170 \\
\hline
\end{tabular}

Fonte: Dados da pesquisa.

A rede de colaboração científica da SBM é composta por 1279 nós que se relacionam 3347 vezes (arestas), organizando-se em 199 clusters, do que resulta uma média de 6,4 autores por cluster calculado. O número médio de conexões entre os nós é 2,617, porém, quando essas conexões são ponderadas pelos pesos das arestas, esse valor sobe 0,08 ponto $(2,7)$, indicando que 102 arestas se repetiram entre os nós.

Na SSCP, a rede é composta por um percentual de nós e um percentual de arestas, respectivamente, $45 \%$ (703) e 64\% (1202) menores do que aqueles encontrados na SBM. Essa relação impacta diretamente o grau médio da rede, que tiveram uma média de 1,71 aresta por nó. Quando observado o grau ponderado, os valores ampliam-se em 0,06 ponto $(1,77)$, que é uma proporção semelhante ao crescimento identificado na SBM; porém, no caso da SSCP, indica a repetição de 72 conexões entre os autores.

Esse panorama demonstra que, na cultura científica da SSCP, o trabalho colaborativo, ainda que se observe um processo de crescimento, ainda é pouco consolidado, pois a maior parte dos pesquisadores que compõem esse subcampo de investigação tem colaboração estabelecida com menos de dois autores, enquanto na SBM esse número é superior a 2,5.

Quando analisadas as densidades e os componentes conectados nas redes, as duas subáreas apresentam um perfil semelhante: as redes são amplamente fragmentadas e a densidade das redes é muito baixa, o que significa que a rede necessitaria produzir um elevado número de arestas para estar completa, reduzindo o número de componentes conectados. 


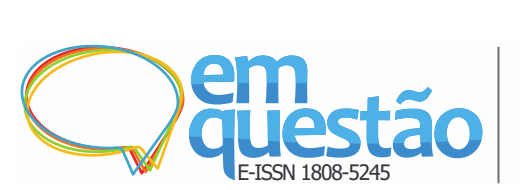

De acordo com Newman (2001), as redes contêm conglomerados ou comunidades científicas locais formadas por um número elevado de indivíduos que se conhecem mais que os demais. Isso significa que os autores tendem a criar grupos coesos e densos e redes cada vez menos fragmentadas. Para Newman (2001), esse é um processo desejado na ciência, uma vez que favorece o intercâmbio de técnicas e conhecimentos entre uma comunidade altamente especializada, favorecendo, assim, o desenvolvimento de um campo.

As existência de redes fragmentadas nas duas subáreas da Educação Física pode ser explicada por alguns fatores que podem existir de maneira isolada ou complementar, quais sejam:

a) a ausência de projetos de pesquisa interinstitucionais;

b) a característica multidisciplinar das duas subáreas, que faz com que exista uma ampla diversidade de temas e objetos, o que produz comunidades especializadas que não dialogam;

c) o isolamento do trabalho de um elevado quantitativo de grupos de pesquisa;

d) corolário desse último, as disputas entre grupos de pesquisa pelos espaços de autoridade no campo científico, de maneira que as colaborações se encerrem no interior do próprio grupo.

A análise do coeficiente de clusterização médio permite algumas inferências complementares sobre esse cenário. De acordo com Barabási (2009), esse coeficiente versa, por um lado, sobre o crescimento das redes e do número de vértices e, por outro, sobre a probabilidade de que um novo vértice se conecte a outro vértice existente na rede de maneira não aleatória. Essa probabilidade exibe uma propriedade de ligação preferencial, ou seja, é altamente provável que dois nós relacionados a um terceiro estejam também relacionados entre si.

O perfil identificado nas redes de colaboração científica tanto na SBM quanto na SSCP se aproxima daquilo que Watts e Strogatz (1998) caracterizaram como redes do tipo small worlds (mundos pequenos), nas quais os nós formam agrupamentos mais ou menos complexos, porém esses agrupamentos não estabelecem conexões entre si, uma vez que, nesse tipo de 


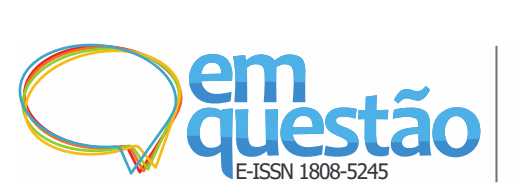

Redes de colaboração em Educação Física: comparação entre a subárea Biodinâmica do Movimento e a subárea Sociocultural e Pedagógica

Felipe Ferreira Barros Carneiro, Amarilio Ferreira Neto e Wagner dos Santos

rede, o coeficiente médio de caminho costuma ser inferior a seis (no caso, 2,048 para a SBM e 3,949 para a SSCP).

A estrutura epistemológica que fundamenta o conceito de small world vai no sentido contrário ao do argumento de que o processo de desenvolvimento científico ocorre segundo uma lógica de fragmentação, sem interface entre os diferentes grupos. Considera-se que há ligações entre os autores que mantêm um nível de coesão para que as atividades se tornem familiares entre os membros dos diferentes grupos (UZZI; SPIRO, 2005). Assim, formam-se subconjuntos coesos de autores que apresentam conexões relativamente frequentes (WASSERMAN; FAUST, 1994), o que lhes confere uma subcultura científica própria (SCOTT, 2000).

Para determinar efetivamente se uma rede é do tipo small world, os cálculos foram efetuados conforme o modelo proposto por Newman, Strogatz e Watts (2001), que consideram a relação que envolve o quociente dos valores observados nos coeficientes de clusterização médio e de caminho médio multiplicado pelo quociente dos valores esperados desses mesmos coeficientes. O modelo de Newman, Strogatz e Watts (2001) toma como referência os valores dessas métricas existentes nos componentes gigantes das redes.

Figura 3 - Componentes gigantes das redes de colaboração científica da SBM e da SSCP no período de 2005 a 2016

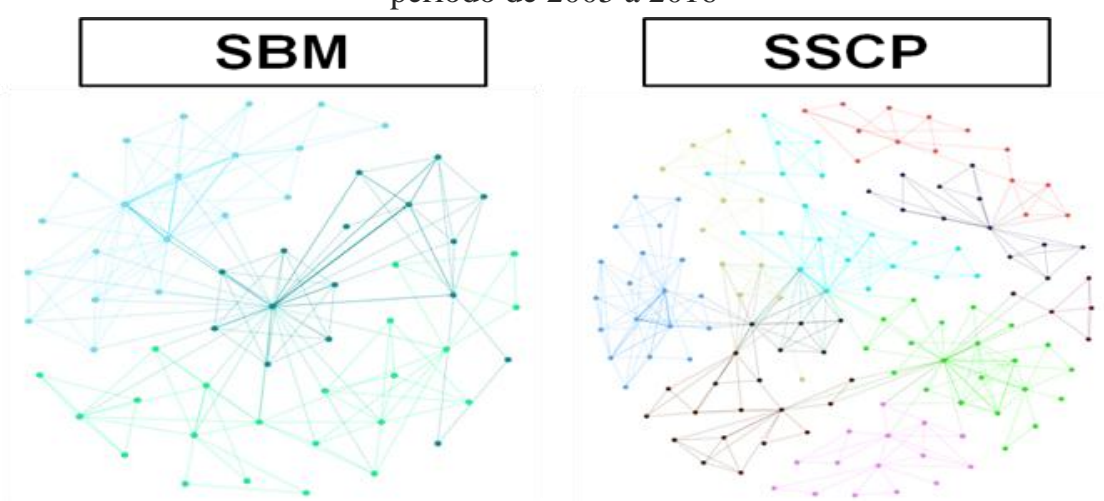

Fonte: Dados da pesquisa. 
Tabela 2 - Características dos componentes gigantes das redes de colaboração científica da SBM e da SSCP para cálculo de small worlds

\begin{tabular}{lcc}
\multicolumn{1}{c}{ Valores } & SBM & SSCP \\
\hline Número de nós & 58 & 140 \\
Número de arestas & 173 & 359 \\
Grau médio & 2,98 & 2,56 \\
Coeficiente de clusterização médio & 0,872 & 0,865 \\
Coeficiente médio de caminho & 2,606 & 4,315 \\
\hline
\end{tabular}

Fonte: Dados da pesquisa.

O coeficiente de clusterização esperado é obtido pela seguinte fórmula:

$$
\text { CDesperado }=k / n
$$

Onde:

$C \Delta$ esperado $=$ coeficiente de clusterização esperado

$$
\begin{gathered}
n=\text { número de nós } \\
k=\text { grau médio }
\end{gathered}
$$

Logo, para a SBM:

$$
\text { C } \Delta \text { esperado }=0,051 \text { e } 0,018
$$

e para a SSCP:

$$
C \Delta \text { esperado }=0,018
$$

Já o coeficiente de caminho médio esperado é calculado por:

$$
\text { Lesperado }=\ln (n) / \ln (k)
$$

Onde:

Lesperado $=$ coeficiente de caminho médio esperado

$\ln (n)=$ logaritmo natural do número de nós

$\ln (k)=$ logaritmo natural do grau médio

Logo, para a SBM:

$$
\text { Lesperado }=3,725,26
$$

e para a SSCP:

$$
\text { Lesperado }=5,26
$$

Para conceber o cálculo dos small worlds ( $\theta)$, Newman, Strogatz e Watts (2001) estabeleceram que:

$$
\theta=\left(\frac{\text { CDobservado }}{\text { Lobservado }}\right) \cdot\left(\frac{\text { Lesperado }}{\text { CDesperado }}\right)
$$


Onde:

$C \Delta o b s e r v a d o=$ coeficiente de clusterização médio observado

Lobservado $=$ coeficiente de caminho médio observado

$\mathrm{e}$

Se $\theta>1$, então existem small worlds ou, se $\theta<1$, então não existem small worlds.

Logo, para a SBM:

$\theta=24,41$

e para a SSCP:

$\theta=58,44$

Conforme pode ser verificado, no caso das redes da SBM e da SSCP, as estatísticas da topologia do componente gigante condizem com os indicadores que Newman, Strogatz e Watts (2001) afirmaram que caracterizavam redes do tipo small worlds $(\theta>1)$.

O valor de $\theta$ encontrado na SBM da Educação Física pode ser considerado pouco expressivo quando comparado a estudos desenvolvidos em outros campos científicos brasileiros, como os de Gestão de Operações $(\theta=$ 209,5), identificados por Martins e colaboradores (2012), no período de 1995 a 2010, e no de Finanças $(\theta=37,7)$, encontrados por Mendes-da-Silva, Onusic e Giglio (2013) entre os anos de 2003 e 2012.

Já o valor de $\theta$ da SSCP é mais significativo que da outra subárea, no que diz respeito à existência de small worlds nas redes de colaboração científica desse campo de investigação. No entanto, são intermediários quando comparados aos das áreas de Finanças e de Gestão de Operações.

Esse panorama reforça a ideia de que o trabalho colaborativo nas duas subáreas do campo de Educação Física tem se desenvolvido de maneira fragmentada em razão da formação de grupos de pesquisadores altamente coesos que atuam em nichos temáticos altamente especializados, cada qual desenvolvendo seus grupos e projetos em seus temas e objetos de estudo específicos. 


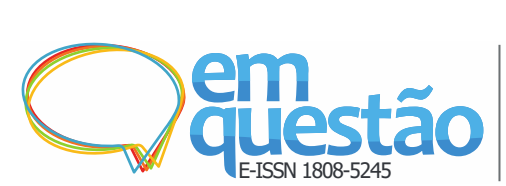

Quando analisado o quantitativo de clusters formados nas redes de colaboração científica, algumas diferenças se salientam entre a SBM e a SSCP. Ainda que as subáreas se desenvolvam de maneira fragmentada, é possível identificar agrupamentos compostos por um elevado quantitativo de pesquisadores conectados.

Porém, conforme pode ser conferido na Tabela 1, o número de clusters formados pelos pesquisadores da SBM é maior do que o de clusters formados pela SSCP. Esse é o cenário esperado, uma vez que a SBM reúne um quantitativo maior do que a SSCP, quando considerado o número de autores que assinam os artigos analisados.

No entanto, a Tabela 3 (a seguir) indica que a diferença entre as redes de colaboração científica das subáreas vai além do número de clusters identificados. Existe, também, uma relação distinta na maneira como esses agrupamentos se conectam e produzem conhecimento.

Tabela 3 - Relação de clusters com N autores nas redes de colaboração científica da SBM e da

\begin{tabular}{ccccc} 
& \multicolumn{2}{c}{ SSCP } & \multicolumn{1}{c}{ SSCP } \\
Autores $(\mathbf{N})$ & Clusters com N autores & \% & Clusters com N autores & \% \\
\hline $\mathbf{2 5 - 3 0}$ & 4 & 2 & - & 0 \\
$\mathbf{2 0 - 2 4}$ & 3 & 1,5 & 5 & 2,9 \\
$\mathbf{1 5 - 1 9}$ & 8 & 4 & 2 & 1,2 \\
$\mathbf{1 0 - 1 4}$ & 14 & 7 & 9 & 5,3 \\
$\mathbf{5 - 9}$ & 86 & 43,2 & 33 & 19,4 \\
$\mathbf{N}<\mathbf{5}$ & 84 & 42,3 & 121 & 71,2 \\
Total & 199 & 100 & 170 & 100 \\
\hline
\end{tabular}

Fonte: Dados da pesquisa.

A Tabela 3 demonstra que os clusters formados pelos pesquisadores da SBM são maiores do que os encontrados na rede de colaboração da SSCP. Além disso, o quantitativo de agrupamentos com elevado número de autores também é maior na SBM, na qual 14\% dos agrupamentos (29) são formados por dez ou mais nós e 57,7\% (115) são compostos por um quantitativo superior a quatro pesquisadores.

Já no caso da SSCP, mais de $70 \%$ dos clusters são formados por menos de cinco autores e 9,4\% (16) são compostos por mais de dez nós, e não há nenhum cluster com mais de 24 cientistas colaborando entre si. 


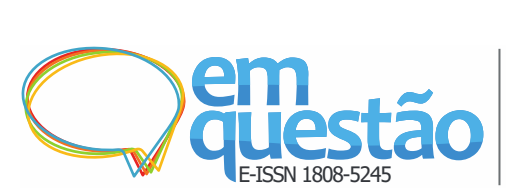

Essas características demonstram que, além de a média de autores por artigo na SBM ser maior, também existe uma conexão entre os objetos e temas investigados em diferentes artigos. Isso indica, nessa subárea, a existência de projetos de pesquisa de longo prazo que envolvem sujeitos em diferentes estágios de formação, produzindo uma diversidade de nós, mas com baixa repetição entre as arestas, o que explicaria a pouca diferença entre os valores encontrados no grau médio e no grau médio ponderado.

Na SSCP, esse fenômeno também parece ocorrer, porém em menor escala, sendo possível inferir que nessa subárea predominam os projetos de pesquisa com duração menor e com pouca manutenção de temas, objetos e linhas de pesquisa. Assim, ainda que envolvam cientistas com diferentes níveis de especialização acadêmica, as conexões são fragmentadas e pouco se repetem.

O formato apresentado nas redes de colaboração científica das duas subáreas impacta diretamente a produtividade dos pesquisadores. Lee e Bozeman (2005) argumentam que, em algumas áreas, o crescimento do trabalho colaborativo está associado ao aumento da produtividade. Isso ocorre, provavelmente, porque os pesquisadores mais centrais tendem a ser mais experientes no processo de produção do conhecimento, o que pode indicar maior capacidade de gerenciamento de recursos humanos.

Nesses termos, ainda que o trabalho colaborativo possa sofrer críticas nos estudos de tradição humanística na área de Educação Física, a produtividade dos autores que praticam a ciência na SSCP têm sido mais ampliada do que a daqueles que atuam na SBM.

A Figura 4 apresenta as redes de colaboração científica da SBM nos períodos compreendidos entre os anos de 2005-2008, 2009-2012 e 2013-2016, bem como as respectivas análises de seus componentes gigantes. Cabe ressaltar que muitas vezes os clusters não se repetem entre os períodos, indicando que, nas distintas periodizações, o corpus de análise não foi sempre composto dos mesmos autores. Esse fato também afeta diretamente o tamanho e a complexidade dos componentes gigantes existentes nos diferentes grafos.

As informações respectivas à Figura 4 encontram-se representadas na Tabela 4. 
Figura 4 - Alterações nas redes de colaboração dos pesquisadores da SBM nos períodos de 2005-2008, 2009-2012 e 2013-2016
2005-2008
2009-2012
2013-2016

Fonte: Dados da pesquisa.

Tabela 4 - Tabela de alterações ocorridas nas redes de colaboração científica da SBM nos períodos de 2005-2008, 2009-2012 e 2013-2016

\begin{tabular}{lccc} 
Indicadores & $\mathbf{2 0 0 5 - 2 0 0 8}$ & $\mathbf{2 0 0 9 - 2 0 1 2}$ & $\mathbf{2 0 1 3 - 2 0 1 6}$ \\
\hline Número de nós & 354 & 431 & 494 \\
Número de arestas & 800 & 957 & 1208 \\
Grau médio & 2,26 & 2,22 & 2,445 \\
Densidade da rede & 0,013 & 0,010 & 0,010 \\
Componentes conectados & 79 & 94 & 93 \\
Diâmetro da rede & 4 & 5 & 3 \\
Coeficiente de clusterização médio & 0,97 & 0,97 & 0,99 \\
Coeficiente médio de caminho & 1,49 & 1,28 & 1,16 \\
\hline \multicolumn{4}{c}{ Topologia de componente gigante } \\
\hline Número de nós & 32 & 15 & 14 \\
Número de arestas & 96 & 53 & 3,71 \\
Grau médio & 3 & 3,53 & 0,959 \\
Coeficiente de clusterização médio & 0,895 & 0,909 & 1,923 \\
Coeficiente médio de caminho & 2,113 & 1,495 & $\theta>1(3,86)$ \\
Coeficiente de small world & $\theta>1(14,7)$ & $\theta>1(5,7)$ & \\
\hline
\end{tabular}

Fonte: Dados da pesquisa.

Quando analisado o número de nós que compõem as redes da SBM, observa-se que nos três períodos houve um crescimento no quantitativo de pesquisadores que assinaram os trabalhos. Desse modo, o período de 2009-2012 reuniu $21,75 \%$ (77) autores a mais que o período de 2005-2008, e o período de 2013-2016 apresentou um crescimento de 14,62\% (63 autores) em relação ao período anterior, no que diz respeito ao número de nós.

Esse desenvolvimento também é acompanhado pelo aumento no número de arestas e da colaboração. Porém, num primeiro momento, o grau médio não acompanha esse crescimento, pois, ao mesmo tempo que se amplia a quantidade 


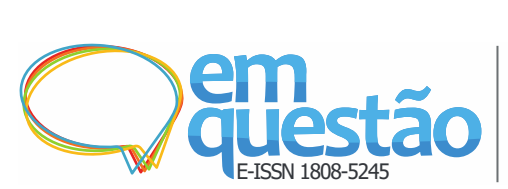

de nós, a densidade de rede diminui, ao passo que cresce o número de componentes conectados.

Além disso, no período de 2009-2012, o quantitativo de arestas aumentou $16,4 \%$ (157), ou seja, teve um crescimento menor do que o número de autores, o que favoreceu a manutenção do coeficiente de clusterização médio em relação ao período de 2005-2008. Isso indica que entre 2009-2012 o crescimento da comunidade científica não foi acompanhado pela ampliação do trabalho colaborativo entre grupos de pesquisadores diversos.

Já no período de 2013-2016, o número de arestas cresceu 26,23\% (251) em relação a 2009-2012, apresentando um aumento superior ao registrado no número de nós entre os mesmos períodos. Assim, o período de 2013-2016 perfez o maior grau médio dos três períodos. O número de componentes conectados nesse período não cresceu, denotando menor fragmentação da rede em relação à quantidade de autores quando comparadas as temporalidades situadas entre os anos de 2005 a 2012. Porém, por mais que tenham se formado mais clusters, a relação entre os autores permanece inalterada (densidade).

Nos anos de 2005-2008, 2009-2012 e 2013-2016, a colaboração científica da SBM apresentou um perfil de redes do tipo small world $(\theta>1)$, denotando que em todos os períodos, a ciência praticada nessa subárea se desenvolveu com pouca articulação entre os diferentes grupos de pesquisadores, uma vez que tem avançado a partir da elevada coesão de coletivos de pesquisadores especializados.

Outro elemento que explica a formação de small worlds nas redes de colaboração científica é a localização geográfica dos pesquisadores, pois é mais frequente a formação de parcerias entre cientistas que desenvolvam suas investigações nos mesmos estados e/ou macrorregiões do país. A distância favorece, assim, a fragmentação da rede e a formação de clusters que apresentam relações mais duradouras e que passam a constituir uma subcultura científica familiar entre si (SCOTT, 2000).

A Figura 5 apresenta as alterações que ocorreram nas redes de colaboração científica da SSCP nos períodos organizados de 2005 a 2016. Assim como os grafos da Figura 4, os clusters e componentes gigantes não 


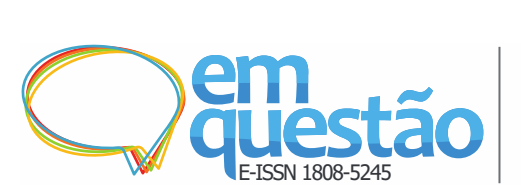

Redes de colaboração em Educação Física: comparação entre a subárea Biodinâmica do Movimento e a subárea Sociocultural e Pedagógica

Felipe Ferreira Barros Carneiro, Amarilio Ferreira Neto e Wagner dos Santos

necessariamente estão conectados entre os períodos de 2005-2008, 2009-2012 e 2013-2016. Desse modo, nós e arestas existentes em um período podem não estar representados na temporalidade anterior ou subsequente.

Dito isso, ao se analisar a Figura 5, observa-se que o crescimento da colaboração científica da SSCP entre os distintos períodos apresentou um fluxo de ampliação do número de pesquisadores e de interações entre eles, diferentemente do que se observou quanto à SBM.

Figura 5 - Alterações nas redes de colaboração dos pesquisadores da SSCP nos períodos de 2005-2008, 2009-2012 e 2013-2016

\begin{tabular}{|l|l|}
\hline $2005-2008$ & $2009-2012$ \\
$2013-2016$ \\
\hline
\end{tabular}

Fonte: Dados da pesquisa.

Tabela 5 - Tabela de alterações ocorridas nas redes de colaboração científica da SSCP nos períodos de 2005-2008, 2009-2012 e 2013-2016

Indicadores

\begin{tabular}{lccc}
\multicolumn{1}{c}{ Indicadores } & $\mathbf{2 0 0 5 - 2 0 0 8}$ & $\mathbf{2 0 0 9 - 2 0 1 2}$ & $\mathbf{2 0 1 3 - 2 0 1 6}$ \\
\hline Número de nós & 212 & 270 & 221 \\
Número de arestas & 259 & 354 & 323 \\
Grau médio & 1,222 & 1,311 & 1,462 \\
Densidade da rede & 0,012 & 0,01 & 0,013 \\
Componentes conectados & 78 & 79 & 70 \\
Diâmetro da rede & 3 & 7 & 3 \\
Coeficiente de clusterização médio & 0,939 & 0,915 & 0,961 \\
Coeficiente médio de caminho & 1,22 & 3,38 & 1,25 \\
\hline \multicolumn{4}{c}{ Topologia de componente gigante } \\
\hline Número de nós & 9 & 54 & 15 \\
Número de arestas & 36 & 100 & 3,6 \\
Grau médio & 4 & 1,852 & 0,933 \\
Coeficiente de clusterização médio & 1 & 0,855 & 1,657 \\
Coeficiente médio de caminho & 1 & 3,846 & $\theta>1(4,86)$ \\
Coeficiente de small world & $\theta>1(3,6)$ & $\theta>1(41,6)$ & \\
\hline
\end{tabular}

2009-2012 
Fonte: Dados da pesquisa.

Conforme a Tabela 5, no período de 2009-2012, o quantitativo de autores que assinaram os trabalhos cresceu 27,4\% (58) em relação ao período anterior, crescimento acompanhado pelo aumento no número de arestas em $36,7 \%$ (95), fazendo com que o grau médio passasse de 1,22 para 1,31. Porém, ao mesmo tempo, a rede se tornou menos densa e com menor coeficiente de clusterização médio, indicando que o crescimento da rede e do trabalho colaborativo não foi seguido pela ampliação das relações entre os pesquisadores de agrupamentos distintos.

No período de 2013-2016, ocorreu uma redução na quantidade de nós que formam a rede em $18,15 \%$ (49). No entanto, ainda que a quantidade de arestas também tenha diminuído em relação ao período anterior, o grau médio aumentou, indicando um crescimento na colaboração científica nos estudos sociopedagógicos. Além disso, a relação entre grupos de cientistas diversos ampliou-se, pois na rede desse período há mais clusters e menos componentes conectados do que em toda a série histórica.

A Tabela 5 indica que, assim como na SBM, as redes de colaboração científica formadas na SSCP nos períodos de 2005-2008, 2009-2012 e 20132016 também se caracterizaram como small worlds, desenvolvendo-se por meio de grupos de pesquisadores isolados em nichos científicos especializados que pouco se comunicam.

As análises a seguir permitem compreender a evolução da colaboração científica na SBM e na SSCP por meio do entendimento dos processos de crescimento e desenvolvimento da comunidade científica nos anos de 2005 a 2016.

A Figura 6 apresenta a ampliação cumulativa das redes de colaboração científica da SBM compreendidos nos seguintes períodos temporais: 2005-2008, 2005-2012 e 2005-2016. Também são representados os grafos com a topologia de componente gigante e seu processo de crescimento acumulado nos respectivos momentos da série histórica.

As informações relativas a esses grafos foram organizadas na Tabela 6 . 


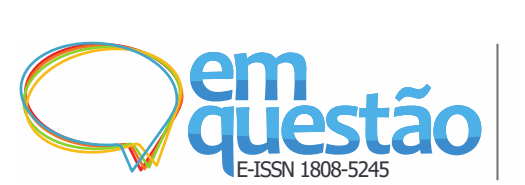

Redes de colaboração em Educação Física: comparação entre a subárea Biodinâmica do Movimento e a subárea Sociocultural e Pedagógica

Felipe Ferreira Barros Carneiro, Amarílio Ferreira Neto e Wagner dos Santos

Figura 6 - Evolução das redes de colaboração da SBM entre os períodos de 2005 a 2016

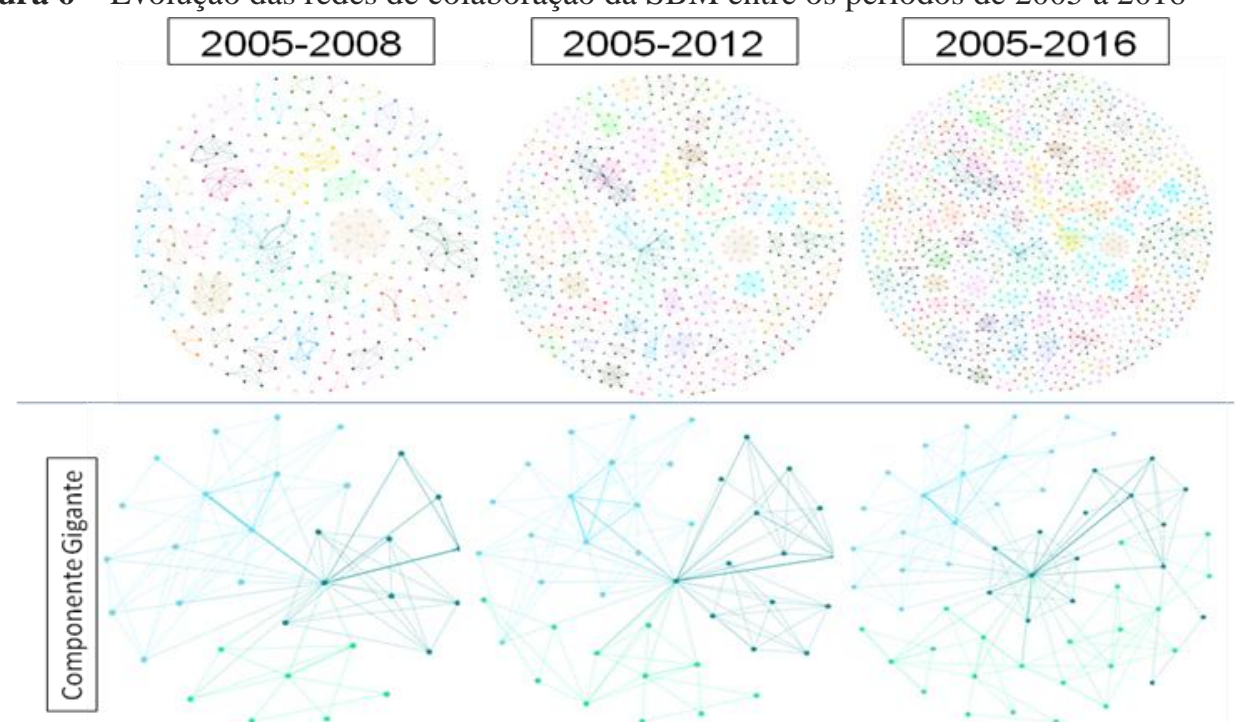

Fonte: Dados da pesquisa.

Tabela 6 - Tabela de evolução dos índices referentes às redes de colaboração científica da SBM

\begin{tabular}{lccc}
\multicolumn{1}{c}{ Indicadores } & nos períodos de 2005 a 2016 & \\
& $\mathbf{2 0 0 5 - 2 0 0 8}$ & $\mathbf{2 0 0 5 - 2 0 1 2}$ & $\mathbf{2 0 0 5 - 2 0 1 6}$ \\
\hline Número de nós & 354 & 785 & 1279 \\
Número de arestas & 800 & 1908 & 3347 \\
Grau médio & 2,26 & 2,431 & 2,617 \\
Densidade da rede & 0,013 & 0,006 & 0,004 \\
Componentes conectados & 79 & 139 & 195 \\
Diâmetro da rede & 4 & 5 & 6 \\
Coeficiente de clusterização médio & 0,97 & 0,962 & 0,961 \\
Coeficiente médio de caminho & 1,492 & 1,733 & 2,048 \\
\hline \multicolumn{2}{c}{ Topologia de componente gigante } \\
\hline Número de nós & 32 & 42 & 58 \\
Número de arestas & 96 & 131 & 173 \\
Grau médio & 3 & 3,119 & 2,98 \\
Coeficiente de clusterização médio & 0,895 & 0,886 & 0,872 \\
Coeficiente médio de caminho & 2,113 & 2,262 & 2,606 \\
Coeficiente de small world & $\theta>1(14,7)$ & $\theta>1(15,1)$ & $\theta>1(24,41)$ \\
\hline
\end{tabular}

Fonte: Dados da pesquisa.

A Tabela 6 demonstra que o crescimento acumulado da rede, no que diz respeito à quantidade de nós e arestas, produziu também um aumento cumulativo no grau médio, indicando que alguns nós que já existiam na rede em períodos anteriores produziram relações de colaboração com aqueles que passaram a compor o grafo.

No entanto, esse processo foi acompanhado da redução da densidade da rede e a consequente ampliação do número de componentes conectados, formando uma rede cada vez mais fragmentada e com menos relações entre os diferentes clusters. Além disso, o coeficiente de clusterização médio apresenta 


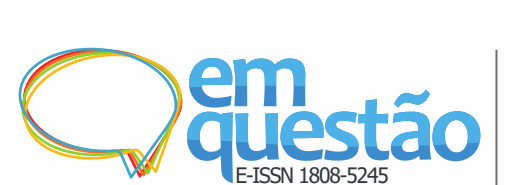

uma redução gradativa com o passar dos anos, o que pode significar que as novas relações estabelecidas entre os autores mais antigos da rede pouco têm produzido conexões entre os agrupamentos já existentes.

A indicação da existência de small worlds é positiva para toda a série histórica. Nesse sentido, quando analisado o componente gigante, nota-se um desenvolvimento contínuo nos três períodos, tanto no número de nós, quanto no de arestas. O crescimento linear acumulado observado nesse elemento topológico é proporcionalmente acompanhado pela ampliação do indicador de small worlds, que, gradativamente, tem se tornado cada vez mais forte na SBM.

Chama a atenção o fato de os clusters que formam esses componentes gigantes serem os mesmos nos três períodos, indicando a existência de grupos de pesquisadores que arregimentam novos nós, tornando suas relações mais coesas e complexas durante toda a série histórica, formando e renovando quadros de cientistas e potencializando o desenvolvimento de uma área de conhecimento especializado nos temas, objetos, métodos e técnicas da ciência praticada na SBM.

No caso da SSCP, conforme a Figura 7 demonstra, também se observa um desenvolvimento acumulativo constante em todos os anos de 2005 a 2016, de modo que as redes de colaboração científica crescem e se tornam sistematicamente mais complexas.

Figura 7 - Evolução das redes de colaboração da SSCP entre os períodos de 2005 a 2016

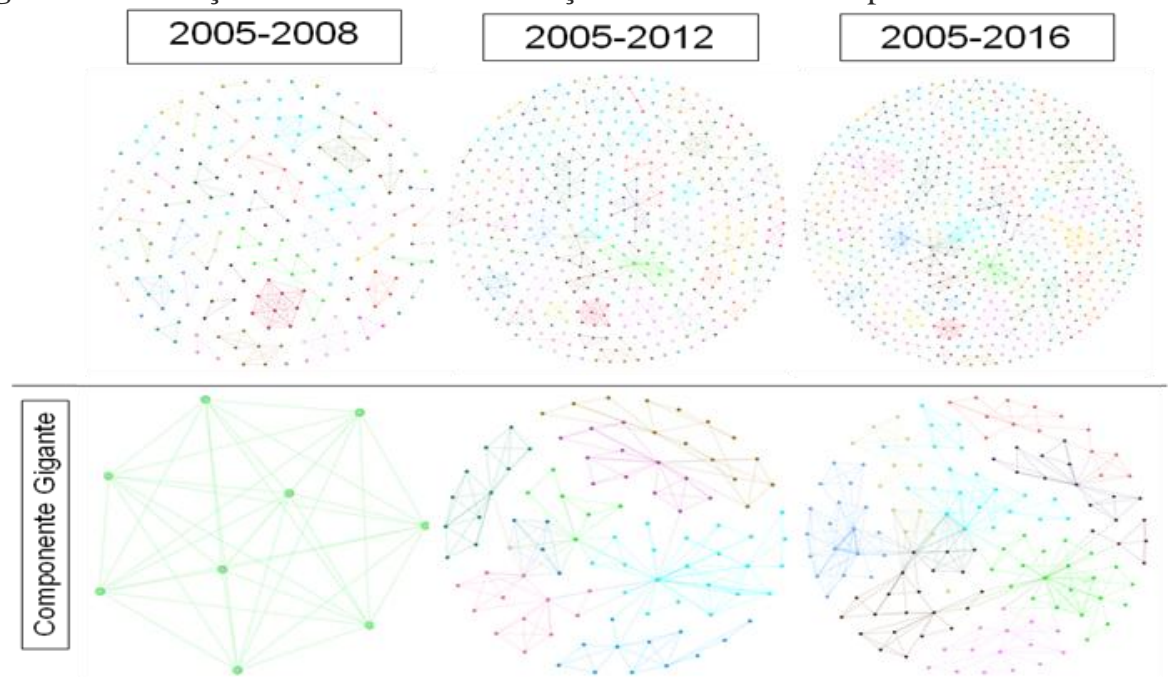

Fonte: Dados da pesquisa. 


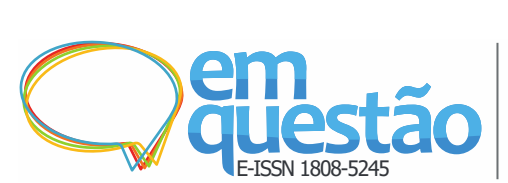

Redes de colaboração em Educação Física: comparação entre a subárea Biodinâmica do Movimento e a subárea

Sociocultural e Pedagógica

Felipe Ferreira Barros Carneiro, Amarílio Ferreira Neto e Wagner dos Santos

Tabela 7 - Tabela de evolução dos índices referentes às redes de colaboração científica da SSCP nos períodos de 2005 a 2016

Indicadores 2005-2008

2005-2012

2005-2016

\begin{tabular}{lccc}
\hline Número de nós & 212 & 482 & 703 \\
Número de arestas & 259 & 730 & 1202 \\
Grau médio & 1,222 & 1,515 & 1,71 \\
Densidade da rede & 0,012 & 0,006 & 0,005 \\
Componentes conectados & 78 & 125 & 161 \\
Diâmetro da rede & 3 & 10 & 10 \\
Coeficiente de clusterização médio & 0,939 & 0,906 & 0,907 \\
Coeficiente médio de caminho & 1,223 & 3,913 & 3,949 \\
\hline \multicolumn{4}{c}{ Topologia de componente gigante } \\
\hline Número de nós & 9 & 94 & 140 \\
Número de arestas & 36 & 209 & 359 \\
Grau médio & 4 & 2,223 & 2,56 \\
Coeficiente de clusterização médio & 1 & 0,877 & 0,865 \\
Coeficiente médio de caminho & 1 & 4,174 & 4,315 \\
Coeficiente de small world & $\theta>1(3,6)$ & $\theta>1(49,6)$ & $\theta>1(58,44)$ \\
\hline
\end{tabular}

Fonte: Dados da pesquisa.

Conforme a Tabela 7 revela, a rede de colaboração científica da SSCP também apresenta um crescimento no número de nós e de arestas em todos os períodos analisados, sendo acompanhado pela elevação do grau médio da colaboração praticada pelos autores que compõem os grafos.

No entanto, assim como na SBM, o aumento do número de nós e arestas foi seguido por uma redução das densidades das redes, indicando uma diminuição das relações estabelecidas entre os autores. Ao mesmo tempo, observa-se um processo de fragmentação crescente em razão do aumento do número de componentes conectados no grafo.

No desenvolvimento da rede da SSCP, o coeficiente de clusterização médio também seguiu uma tendência de redução, denotando que os autores que formam os agrupamentos têm produzido poucas novas relações com os clusters já existentes nas redes. Esse cenário indica que os grupos formados pelos autores ganham em complexidade e coesão nas relações científicas, mas pouco dialogam e colaboram entre si.

Quando analisada a topologia dos componentes gigantes formados nas redes da SSCP de 2005 a 2016, nota-se um crescimento expressivo no quantitativo de nós e arestas existentes entre 2005 e 2008 em relação ao acumulado até o ano de 2012 e depois até o último ano que compõe o período estudado. 


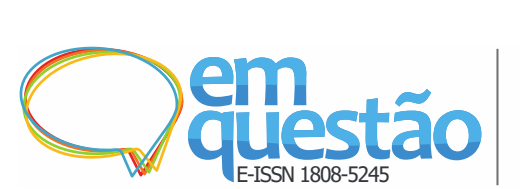

Redes de colaboração em Educação Física: comparação

entre a subárea Biodinâmica do Movimento e a subárea

Sociocultural e Pedagógica

Felipe Ferreira Barros Carneiro, Amarilio Ferreira Neto e Wagner dos Santos

Além disso, diferentemente do ocorrido na SBM, o componente gigante existente entre 2005-2008 é formado por apenas um cluster, em razão da elevada fragmentação da rede de colaboração científica existente no campo científico da SSCP no período em questão.

Assim, o crescimento da rede, ainda que tenha acumulado um quantitativo significativo de fragmentos, tornou-a proporcionalmente mais coesa, de modo que o número de pesquisadores que passam a formar o componente gigante da SSCP é superior ao daqueles que compõem esse elemento topológico da SBM, tanto em números relativos, quanto em valores absolutos, entre os anos de 2005-2012 e de 2005-2016.

A proporção do número de nós que formam os componentes gigantes das redes da SSCP em relação à rede global (especialmente após 2009) é um elemento importante no aumento das estatísticas que demonstram a existência de small worlds nessa subárea (MENDES-DA-SILVA; ONUSIC; GIGLIO, 2013). Cabe ressaltar que essa característica de rede científica, identificada em todos os períodos, vem se fortalecendo cada vez mais na SSCP.

\section{Conclusões}

As análises e a comparação das redes de colaboração científica da SBM e da SSCP permitiram compreender as características e os impactos do trabalho colaborativo nas duas subáreas da Educação Física.

No que diz respeito ao número de nós (autores) e arestas (colaborações) das redes, a rede da SBM se mostrou significativamente maior do que a da SSCP, indicando que os pesquisadores que formam a comunidade científica que investiga os aspectos biodinâmicos do movimento são mais numerosos e estabelecem mais parcerias do que os da comunidade que se dedica a compreender as relações sociopedagógicas da área de Educação Física.

No entanto, nas duas subáreas, as relações entre os pesquisadores se configuram de maneira transitória ou esporádica, uma vez que as arestas que se repetem correspondem, aproximadamente, a 10\% das colaborações existentes nas redes de cada subárea, denotando uma baixa relação entre os pesquisadores que compõem os grupos representados nas redes de maneira fragmentada. 


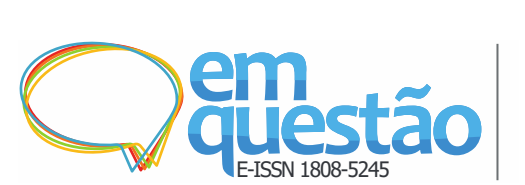

Quando observada a evolução das redes, evidenciou-se que as duas comunidades científicas analisadas se desenvolveram de modo semelhante, apresentando um crescimento em todos os períodos analisados. De 2005 a 2016, a rede de colaboração da SBM cresceu $260 \%$, um crescimento $12,07 \%$ maior que o da SSCP (232\%).

No entanto, quando analisada a topologia de componente gigante, a SSCP cresceu de maneira expressiva em comparação à SBM, indicando o fortalecimento de grupos de pesquisas coesos produzindo conhecimento dentro de uma determinada subcultura científica, fortalecendo as bases do conhecimento que confere sustentação epistemológica aos estudos dessa subárea.

Tanto na SSCP quanto na SBM, as redes de colaboração científica se caracterizaram como small worlds, indicando a fragmentação das redes que, possivelmente, ocorre em virtude da multidisciplinaridade da área de Educação Física, bem como de suas subáreas, que se organizam em nichos científicos povoados por pesquisadores altamente especializados em teorias, temas, objetos, métodos e técnicas de pesquisa próprios.

Nesse sentido, é de se esperar que análises que tomem como base as redes de colaboração científica formadas por pesquisadores que investigam temáticas específicas no interior das subáreas sejam mais densas e com um quantitativo menor de fragmentos (componentes conectados).

No que diz respeito aos clusters, as redes da SBM apresentaram um maior volume de agrupamentos compostos por grandes quantidades de autores do que as redes da SSCP, que, por sua vez, tem a maioria dos clusters formados por menos de cinco autores.

\section{Referências}

BARABÁSI, A-L. Linked: a nova ciência dos networks. São Paulo: Leopardo, 2009.

BRACHT, V. Identidade e crise na Educação Física: um enfoque epistemológico. In: BRACHT, V.; CRISÓRIO, R. Educação Física no Brasil e na Argentina: identidade, desafios e perspectivas. São Paulo: Autores Associados, 2003. cap. 1, p. 13-29. 


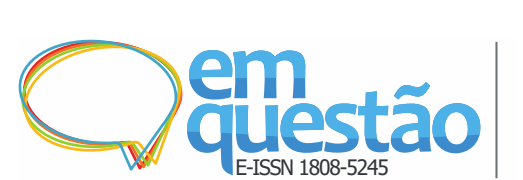

Redes de colaboração em Educação Física: comparação entre a subárea Biodinâmica do Movimento e a subárea Sociocultural e Pedagógica

Felipe Ferreira Barros Carneiro, Amarilio Ferreira Neto e Wagner dos Santos

CAMARGO, A. M. Sociedade em Rede: comunicação científica na nova mídia. Curitiba: Appris, 2016.

CAPES. Critérios de classificação Qualis Área 21 - Educação Física, Fisioterapia, Fonoaudiologia, e Terapia Ocupacional. Brasília, p. 1-7, abr. 2016.

CARNEIRO, F. F. B.; FERREIRA NETO, A.; SANTOS, W. dos. Práticas científicas em educação física: tradições e tensões. Curitiba: CRV, 2015.

CNPQ. Disponível em : <http://www.cnpq.br/web/guest/bolsistas-vigentes/> Acesso em: 15 de fev. de 2017.

CURIEL, E. H. La credibilidad de las redes sociales en el ámbito periodístico. Transinformação, Campinas, v. 27, n. 2, p. 165-171, 2015.

DIGIAMPIETRI, L. A. et al. Análise da rede de relacionamentos dos doutores brasileiros. In: CONGRESSO DA SOCIEDADE BRASILEIRA DE COMPUTAÇÃO, 34., 2014, Brasília. Anais [...]. Brasília: Sociedade Brasileira de Computação, 2014. p. 1-8.

FRUCHTERMAN, T. M. J.; REINGOLD, E. M. Graph Drawing by ForceDirected Placement. Software, [s.l.], v. 21, n. 11, p. 1129-1164, 1991.

HILÁRIO, C. M.; GRÁCIO, M. C. C.; GUIMARÃES, J. A. C. Aspectos éticos da coautoria em publicações científicas. Em Questão, Porto Alegre, v. 24, n. 2, p. 12-36, 2018.

KATZ, J. S.; MARTIN, B. R. What is research collaboration? Research Policy, Amsterdam, v. 26, n. 1, p. 1-18, 1997.

KOKUBUN, E. Avaliação da educação física em debate: esclarecimentos. Revista Brasileira de Pós-Graduação, Brasília, v. 1, n. 2, p. 195-200, 2004.

LARIVIÈRE, V. et al. The place of serials in referencing practices: comparing natural sciences and engineering with social sciences and humanities. Journal of the American Society for Information Science and Technology, Nova Jersey, v. 57, n. 8, p. 997-1004, 2006.

LUIZ, R. R.; MAGNANINI, M. F. O tamanho da amostra em pesquisas epidemiológicas. In: MEDRONHO, R. A.; BLOCH, K. V.; LUIZ, R. R.; WERNECK, G. L. Epidemiologia. São Paulo: Atheneu, 2009. cap. 23, p. 415427.

LEE, S.; BOZEMAN, B. The impact of research collaboration on scientific productivity. Social Studies of Science, Londres, v. 35, n. 5, p. 673-702, 2005. 


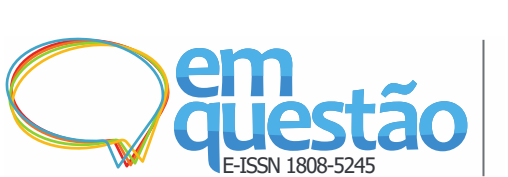

Redes de colaboração em Educação Física: comparação entre a subárea Biodinâmica do Movimento e a subárea Sociocultural e Pedagógica

Felipe Ferreira Barros Carneiro, Amarilio Ferreira Neto e Wagner dos Santos

MANOEL, E. J.; CARVALHO, Y. M. Pós-graduação na Educação Física brasileira: a atração (fatal) para a biodinâmica. Educação e Pesquisa, São Paulo, v. 37, n. 2, p. 389-406, 2011.

MARTINS, M. E. et al. Service's scientific community: a social network analysis (1995-2010). Journal of Service Management, West Yorkshire, v. 23, n. 3, p. 455-469, 2012.

MENDES-DA-SILVA, W.; ONUSIC, L. M.; GIGLIO, E. M. Redes de pesquisadores de finanças no Brasil: um mundo pequeno feito por poucos. Revista de Administração Contemporânea, Rio de Janeiro, v. 17, n. 6, p. 739$763,2013$.

NEWMAN, M. E. J. The structure of scientific collaboration networks. Proceedings of the National Academy of Sciences of the United States of America, Santa Fe, v. 98, n. 2, p. 404-409, 2001.

NEWMAN, M. E. J.; STROGATZ, S. H.; WATTS, D. J. Random graphs with arbitrary degree distributions and their applications. Physical Review E, Maryland, v. 64, n. 2, p. 64-81, 2001.

PINTO, A. L.; GONZALES-AGUILAR, A. Visibilidad de los estudios en análisis de redes sociales en América del Sur: su evolución y métricas de 19902013. Transinformação, Campinas, v. 26, n. 3, p. 253-267, 2014.

SCOTT, J. Social Network Analysis: a handbook. 2nd. ed. London: Sage Publications, 2000.

SIDONE, O. J. G.; HADDAD, E. A.; MENA-CHALCO, J. P. A ciência nas regiões brasileiras: evolução da produção e das redes de colaboração científica. Transinformação, Campinas, v. 28, n. 1, p. 15-32, 2016.

SOUSA, E. S.; FONTENELE, R. E. S. Mapeamento da produção científica internacional sobre Valores Humanos Básicos. Em Questão, Porto Alegre, v. 25, n. 3, p. 214-245, 2019.

TANI, G. Perspectivas da educação física como disciplina acadêmica. In: SIMPÓSIO PAULISTA DE EDUCAÇÃO FÍSICA, 2., 1989, Rio Claro. Anais [...]. Rio Claro: Universidade Estadual Paulista, 1989. p. 2-12.

TODESCHINI, R.; BACCINI, A. Handbook of Bibliometric Indicators: quantitative tools for studying and evaluating research. Weinherim: WileyVHC, 2016.

TORRES, T. Z. G.; MAGNANINI, M. M. F.; LUIZ, R. R. Amostragem. In: MEDRONHO, R. A.; BLOCH, K. V.; LUIZ, R. R.; WERNECK, G. L. Epidemiologia. São Paulo: Atheneu, 2009. cap. 22, p. 403-413. 


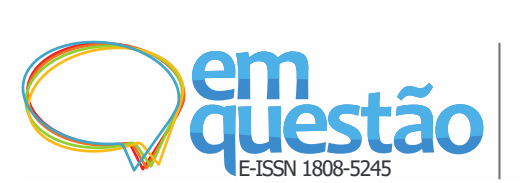

Redes de colaboração em Educação Física: comparação entre a subárea Biodinâmica do Movimento e a subárea Sociocultural e Pedagógica

Felipe Ferreira Barros Carneiro, Amarilio Ferreira Neto e Wagner dos Santos

UZZI, B.; SPIRO, J. Collaboration and creativity: the small world problem. American Journal of Sociology, Chicago, v. 111, n. 2, p. 447-504, 2005.

VANZ, S. A. S.; STUMP, I. R. C. Colaboração científica: revisão teóricoconceitual. Perspectivas em Ciência da Informação, Belo Horionte, v. 15, n. 2, p. 42-55, 2010.

WASSERMAN, S.; FAUST, K. Social Network Analysis: methods and applications. Cambridge: Cambridge University Press, 1994.

WATTS, D. J.; STROGATZ, S. H. Collective dynamics of "smallworld" networks. Nature, Londres, v. 393, p. 440-442, 1998.

\title{
Scientific collaboration networks in the field of Physical \\ Education: a comparative analysis between its subfield of \\ Biomechanics and its sociocultural and pedagogical subfield
}

\begin{abstract}
This study aims to investigate, characterize and analyze the scientific collaboration networks in the field of Physical Education, in order to understand the historical development of the networks of its subfield of Biomechanics and its sociocultural and pedagogical subfield and to compare their features and their impacts on scientific communication. We used 10 scientific journals of each subfield. To produce and to manipulate the networks, we used Endnote X7.5 (to create the database), VOSviewer 1.6.10 (to generate the primary network) and Gephi 0.92 through the Fruchterman-Reingold algorithm. We found that both subfields have been developing in a fragmented way, but through small world networks.
\end{abstract}

Keywords: Physical Education. Scientific netwoks. Scientometrics.

Recebido: 16/12/2019

Aceito: 19/03/2020

\footnotetext{
1 Informações recuperadas por meio da plataforma de busca do CNPq, disponível em http://www.cnpq.br/web/guest/bolsistas-vigentes/ com acesso em 15 de fevereiro de 2017.

2 Peso 5 para bolsista de nível 1A; peso 4 para bolsista de nível 1B; peso 3 para bolsista de nível

1C; peso 2 para bolsista de nível 1D; peso 1 para bolsista de nível 2.
} 